Journal of Mathematical AnAlysis

ISSN: 2217-3412, URL: WWW.ILIRIAS.COM/JMA

Volume 12 Issue 5 (2021), Pages 28-39.

DOI:10.54379/JMA-2021-5-2

\title{
SOME CHARACTERIZATIONS OF DICHOTOMY FOR IMPULSIVE DYNAMIC SYSTEMS
}

\author{
GULNAZ ATTA, AWAIS YOUNUS
}

\begin{abstract}
We study the problem of dichotomy and boundedness for impulsive dynamic equations on arbitrary closed subset of real numbers. The spectral decomposition theorem gives all our main results. The obtained results are fundamentally new, even for the classical case.
\end{abstract}

\section{INTRODUCTION}

Dynamical systems has many applications see, for example ([15, 16, 21]) and references therein. In 1960, Millman et al. [11, began to study impulsive differential systems (IDS). To design the impulsive control protocol, the theory of IDS is a standard mathematical method. Research in IDS has gained momentum ([17, 18, 19]). Such a system provides not only realistic models but also presents many interesting mathematical problems. Wang et al. 20, reviewed and addressed some recent developments of impulsive control theory, fundamental theory on impulsive control systems.

An IDS is described by three components:

(1) A differential system, which governs the state of the system between impulses.

(2) An impulse condition, which models an impulsive jump defined by a jump function at the instant an impulse occurs.

(3) A jump rule, which defines a set of jump events in which the impulse equation is active.

Exponential type dichotomy, which traces back to [12 and generalizes the concept of hyperbolicity from autonomous to nonautonomous linear systems, has been playing an ever more critical role in the study of nonautonomous dynamical systems such as dynamic equations on time scales [13, ordinary differential equations [4, 5, 6, and difference equations [1].

2010 Mathematics Subject Classification. 34A37,34D09,34N05.

Key words and phrases. impulsive dynamic systems; dichotomy; impulsive transition matrix, Spectral decomposition theorem.

(C)2021 Ilirias Research Institute, Prishtinë, Kosovë.

Submitted June 22, 2021. Published September 28, 2021.

Communicated by C. Tunc. 
The study of dichotomy for dynamic equations on time scales is quite recent [22]. There are several approaches in the literature to study dichotomy, one of which is the spectral decomposition approach [22].

The presence of impulses in the dynamic equations on time scales leads to complications with the concept of the solution. One major difficulty is the spectral decomposition theorem for impulsive dynamic systems on time scales. To overcome this difficulty, we generalized the spectral decomposition approach to the impulsive case.

In this paper, we investigate the problem of exponential dichotomy and boundedness of solutions of the following Cauchy problems:

$$
\left\{\begin{array}{l}
a^{\Delta}(x)=A a(x)+f(x) Q b, x \neq x_{k}, \\
\Delta a\left(x_{k}^{+}\right)=I_{k}(a(x)), x=x_{k} \\
a\left(x_{0}\right)=a_{0}
\end{array}\right.
$$

and

$$
\left\{\begin{array}{l}
a^{\Delta}(x)=-A a^{\sigma}(x)+f(x)(I-Q b), x \neq x_{k} \\
\Delta a\left(x_{k}^{+}\right)=I_{k}(a(x)), x=x_{k} \\
a\left(x_{0}\right)=a_{0},
\end{array}\right.
$$

where $\mathbb{T}$ is a time scale, $A$ is a regressive matrix $b$ is non-zero vector in $\mathbb{R}^{m}, f(s)$ is a bounded and right-dense continuous function on $\mathbb{T}, Q$ is a projection on $\mathbb{C}^{m}$ and $I_{k}$ is vector-valued impulsive function defined on $\mathbb{R}^{n}$.

Impulsive dynamic system is an important area of study. There are many qualitative results obtained for above systems [7, 8, 9].

The organization of the paper is as follows: Section 2 presents the basic knowledge of time scale calculus, impulsive linear dynamic systems, including the general definition of impulsive transition matrix and its fundamental properties. Section 3 introduces the concept of dichotomy analysis and criteria for impulsive systems with different impulsive conditions. Finally, concluding remarks and propositions for future works are given.

\section{Preliminaries}

To keep this article self-contained, this section contains some preliminary definitions and the associated notation [2, 3. For a nonempty closed subset of real numbers (time scale) $\mathbb{T}$, let us consider the following operators and step size function:

$$
\begin{aligned}
x^{\sigma} & =\sigma(x):=\inf \{s \in \mathbb{T}: s>x\} \\
x^{\rho} & =\rho(x):=\sup \{s \in \mathbb{T}: s<x\},
\end{aligned}
$$

and

$$
\mu: \mathbb{T} \rightarrow \mathbb{R}^{+}: \mu(x):=x^{\sigma}-x .
$$

It is easy to see that for $x \in \mathbb{T}, x^{\sigma} \geq x$ and $x^{\rho} \leq x$.

Throughout this paper, we always assume that $\sup \mathbb{T}=\infty$ and for any $x \in \mathbb{T}$, let $\mathbb{T}_{(x)}:=[x, \infty) \cap \mathbb{T}$ and $\mathbb{T}_{+}:=\mathbb{T}_{(0)}$.

A function $f: \mathbb{T}_{(x)} \rightarrow \mathbb{R}$ is called right-dense continuous (rd-continuous), if $\lim _{x \rightarrow x_{0}^{-}} f(x)$ exists at left-dense points and $\lim _{x \rightarrow x_{0}^{+}} f(x)=f\left(x_{0}\right)$ at right-dense 
points. The delta derivative of $f$ is defined by

$$
f^{\Delta}(x)=\lim _{\substack{s \rightarrow x \\ s \neq x^{\sigma}}} \frac{f\left(x^{\sigma}\right)-f(s)}{x^{\sigma}-s} .
$$

For a finite dimensional Banach space $\mathbb{X}$ the following spaces appear mostly:

$C_{r d}\left(\mathbb{T}_{(\tau)}, \mathbb{X}\right):=\left\{f: \mathbb{T}_{(\tau)} \rightarrow \mathbb{X} \mid f\right.$ is $r d$-continuous $\}$,

$C_{r d}^{1}\left(\mathbb{T}_{(\tau)}, \mathbb{X}\right):=\left\{f: \mathbb{T}_{(\tau)} \rightarrow \mathbb{X} \mid f \Delta\right.$-derivative is in $\left.C_{r d}\left(\mathbb{T}_{(\tau)}, \mathbb{X}\right)\right\}$,

$\mathcal{R}\left(\mathbb{T}_{(\tau)}, \mathbb{X}\right):=\left\{f \in C_{r d}\left(\mathbb{T}_{(\tau)}, \mathbb{X}\right) \mid\left(I_{\mathbb{X}}+\mu(x) f(x)\right)^{-1}\right.$ exists for all $\left.x \in \mathbb{T}_{(\tau)}\right\}$

$\mathcal{R}_{U}\left(\mathbb{T}_{(\tau)}, \mathbb{C}\right):=\left\{f \in \mathcal{R}\left(\mathbb{T}_{(\tau)}, \mathbb{C}\right) \mid\right.$ If $\exists \alpha$ with $\left.\alpha^{-1} \leq|1+(\mu f)(x)| \forall x \in \mathbb{T}_{(\tau)}\right\}$

$\mathcal{R}^{+}\left(\mathbb{T}_{(\tau)}, \mathbb{R}\right) \quad: \quad=\left\{f \in \mathcal{R}\left(\mathbb{T}_{(\tau)}, \mathbb{R}\right) \mid I_{\mathbb{X}}+\mu(x) f(x)>0\right.$ for all $\left.x \in \mathbb{T}_{(\tau)}\right\}$,

where $\mathbb{X}=\mathbb{R}, \mathbb{C}, \mathbb{R}^{n}, \mathbb{C}^{n}$ or $\mathbb{M}_{n}(\mathbb{R})$ and $I_{\mathbb{X}}$ is corresponding multiplicative identity of $\mathbb{X}$.

It is well known that for $A \in \mathcal{R}\left(\mathbb{T}_{+}, \mathbb{M}_{n}(\mathbb{R})\right)$, the exponential matrix $e_{A}(x, s)$ at initial time $s \in \mathbb{T}_{+}$is the unique solution of the following matrix initial value problem (IVP)

$$
X^{\Delta}=A X, X(x)=I_{\mathbb{M}_{n}(\mathbb{R})}
$$

and $a(x)=e_{A}(x, s) a_{0}, x \geq s$, is the unique solution of IVP

$$
a^{\Delta}=A a, a(x)=a_{0}
$$

and $a(x)=e_{\ominus A^{T}}(x, s) a_{0}, x \geq s$ is the unique solution of the following adjoint IVP

$$
a^{\Delta}=-A^{T} a^{\sigma}, a(s)=a_{0} .
$$

Recently, Zada et. al. 22, proved the following spectral decomposition theorem on time scales:

For $A \in \mathcal{R}\left(\mathbb{T}_{+}, \mathbb{M}_{n}(\mathbb{R})\right)$ and for each $z \in \mathbb{C}^{n} \exists w_{i} \in \mathcal{L}_{i}:=\operatorname{ker}\left(A-\lambda_{i} I\right)^{n_{j}}(i=1,2, \cdots k)$

$$
e_{A}(x, 0) z=e_{A}(x, 0) w_{1}+e_{A}(x, 0) w_{2}+\cdots+e_{A}(x, 0) w_{k}, x \in \mathbb{T} .
$$

Furthermore, if $w_{i}(x):=e_{A}(x, 0) w_{i}$ then $w_{i}(s) \in \operatorname{ker}\left(A-\lambda_{i}\right)^{n_{i}} \forall x \in \mathbb{T}$ and $\exists$ $\mathbb{C}^{n}$-valued polynomials $y_{i}(x)$ with $\operatorname{deg}\left(y_{i}\right) \leq n_{i}-1$ :

$$
w_{i}(x)=e_{\lambda_{i}}(x, 0) y_{i}(x), x \in \mathbb{T} \text { and }(i=1,2, \cdots k) .
$$

It follows that

$$
e_{A}(x, 0) z=e_{\lambda_{1}}(x, 0) y_{1}(x)+e_{\lambda_{2}}(x, 0) y_{2}(x)+\cdots+e_{\lambda_{k}}(x, 0) y_{k}(x), x \in \mathbb{T} .
$$

For $I_{k}\left(a\left(x_{k}\right)\right)=I+B_{k}$, consider the following homogenous linear impulsive dynamic systems

$$
\left\{\begin{array}{l}
a^{\Delta}=A a, x \in \mathbb{T}_{+}, x \neq x_{k} \\
a\left(x_{k}^{+}\right)=\left(I+B_{k}\right) a\left(x_{k}\right), k=1,2, \cdots
\end{array}\right.
$$

where $B_{k} \in M_{n}(\mathbb{R})$, for all $k, A \in \mathcal{R}\left(\mathbb{T}_{+}, M_{n}(\mathbb{R})\right), 0=x_{0}<x_{1}<x_{2}<\cdots<x_{k}<$ $\cdots$, with $\lim _{k \rightarrow \infty} x_{k}=\infty, a\left(x_{k}^{+}\right)$represents the right limit of $a(x)$ at $x=x_{k}$ (with $a\left(x_{k}^{+}\right)=a(x)$ if $x_{k}^{\sigma}>x_{k}$ for all $\left.k\right)$.

Throughout we assumed that, $x_{k}^{\sigma}=x_{k}$ for all $k$.

Corresponding to 2.2 consider the following IVP

$$
\left\{\begin{array}{l}
a^{\Delta}=A a, x \in \mathbb{T}_{+}, x \neq x_{k} \\
a\left(x_{k}^{+}\right)=\left(I+B_{k}\right) a\left(x_{k}\right), k=1,2, \cdots, \\
a\left(\tau^{+}\right)=\eta, \tau \geqslant 0
\end{array}\right.
$$


Let us consider the transition matrix $G_{A}(x, s), 0 \leq s \leq x$, of 2.2 associated with impulsive sequence $\left\{B_{k}, x_{k}\right\}_{k=1}^{\infty}$ is defined as

$$
G_{A}(x, s)= \begin{cases}e_{A}(x, s) & \text { if } x_{k-1} \leq s \leq x \leq x_{k} \\ e_{A}\left(x, x_{k}^{+}\right)\left(I+B_{k}\right) e_{A}\left(x_{k}, s\right) & \text { if } x_{k-1} \leq s<x_{k}<x \leq x_{k+1} \\ e_{A}\left(x, x_{k}^{+}\right)\left[\prod_{s<x_{j} \leq x}\left(I+B_{j}\right) e_{A}\left(x_{j}, x_{j-1}^{+}\right)\right] & \\ \left(I+B_{i}\right) e_{A}\left(x_{i}, s\right), & \text { if } x_{i-1} \leq s<x_{i}<\cdots<x_{k}<x \leq x_{k+1}\end{cases}
$$

Example 2.1. Let us consider the following IVP

$$
\left\{\begin{array}{l}
a^{\Delta}=\left(\begin{array}{cc}
-\lambda_{1} & 0 \\
0 & -\lambda_{2}
\end{array}\right) a, x \in \mathbb{T}_{+}, x \neq x_{2 k} \\
a\left(x_{2 k}^{+}\right)=\left(\begin{array}{cc}
a_{2 k} & 0 \\
0 & b_{2 k}
\end{array}\right) a\left(x_{2 k}\right), k=1,2, \cdots \\
a\left(\tau^{+}\right)=\eta, \tau \geqslant 0
\end{array}\right.
$$

where $A=\left(\begin{array}{cc}-\lambda_{1} & 0 \\ 0 & -\lambda_{2}\end{array}\right),\left(I+B_{k}\right)=\left(\begin{array}{cc}a_{2 k} & 0 \\ 0 & b_{2 k}\end{array}\right), \lambda_{2}>\lambda_{1}>0$ and $x_{k}:=k$.

Then the impulsive transition matrix $G_{A}(x, s), 0 \leq s \leq x$, of (2.4) associated with impulsive sequence $\left\{B_{k}, x_{k}\right\}_{k=1}^{\infty}$ is

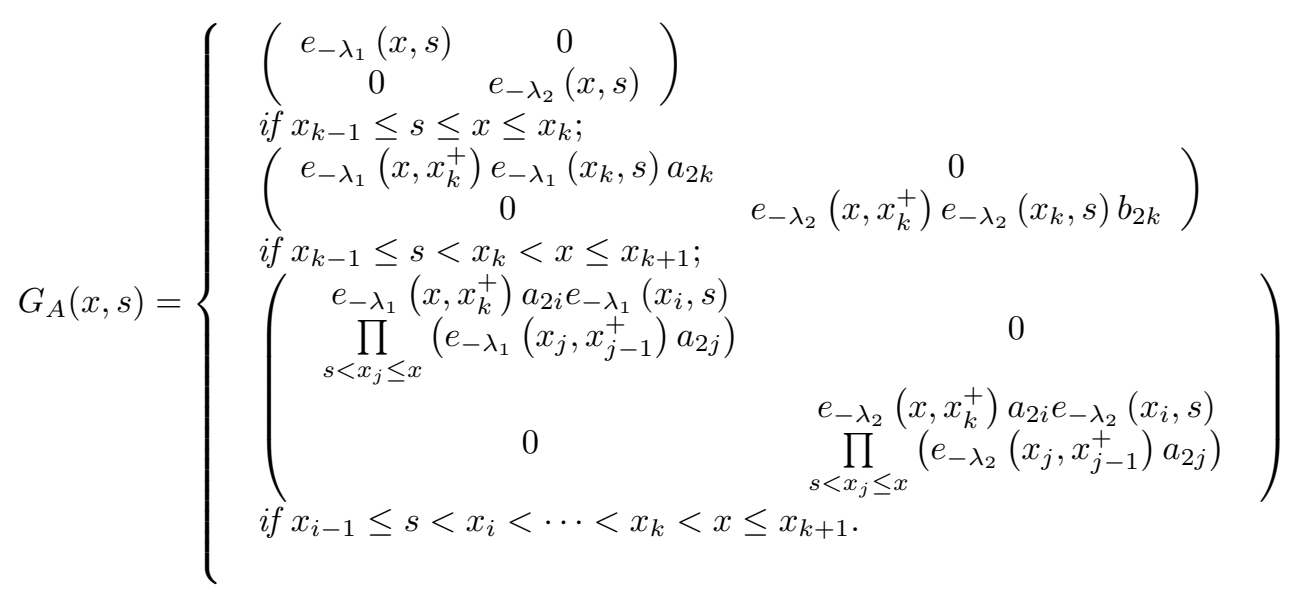

Remark. It is easy to see that spectral decomposition theorem may not holds for $G_{A}(x, s)$. For example, if $B_{k}$ is arbitrary non-diagonal matrix and $A B_{k} \neq B_{k} A$, then $\mathcal{L}_{j}$ is not $G_{A}(x, s)$-invariant subspace of $\mathbb{C}^{n}$.

In this circumstance, we need to modify spectral decomposition theorem for impulsive transition matrix $G_{A}(x, s), 0 \leq s \leq x$, associated with $\left\{B_{k}, x_{k}\right\}_{k=1}^{\infty}$.

$\left(\mathrm{A}_{1}\right)$ : Assume that $A \in \mathcal{R}\left(\mathbb{T}_{+}, M_{n}(\mathbb{R})\right)$ and $B_{k} \in M_{n}(\mathbb{R}), k=1,2, \cdots$.

$\left(\mathrm{A}_{2}\right)$ : Let $\left(\mathrm{A}_{1}\right)$ holds and there exist a positive constant $\theta$ such that $x_{k+1}-x_{k}<\theta$, $k=1,2, \cdots$.

Lemma 2.2. Let $\left(A_{1}\right)$ holds such that $A B_{k}=B_{k} A$ and $\mathcal{L}_{j}$ is $\left(I+B_{k}\right)$-invariant subspace of $\mathbb{C}^{n}$ for each $k=1,2,3, \cdots$. For each $z \in \mathbb{C}^{n} \exists w_{i} \in \mathcal{L}_{i}:=\operatorname{ker}\left(A-\lambda_{i} I\right)^{n_{i}}(i=\overline{1, k})$ :

$$
G_{A}(x, 0) z=G_{A}(x, 0) w_{1}+G_{A}(x, 0) w_{2}+\cdots+G_{A}(x, 0) w_{k}, x \in \mathbb{T} .
$$


Furthermore, if $w_{i}(x):=G_{A}(x, 0) w_{j}$ then $w_{j}(x) \in \operatorname{ker}\left(A-\lambda_{i}\right)^{n_{i}}$ for all $x \in \mathbb{T}$ and $\exists \mathbb{C}^{n}$-valued polynomials $y_{i}(x)$ with $\operatorname{deg}\left(y_{i}\right) \leq n_{i}-1$ :

$$
w_{j}(x)=e_{\lambda_{i}}(x, 0) y_{i}(x), x \in \mathbb{T} \text { and }(i=\overline{1, k}) .
$$

It follows that

$$
G_{A}(x, 0) z=e_{\lambda_{1}}(x, 0) y_{1}(x)+e_{\lambda_{2}}(x, 0) y_{2}(x)+\cdots+e_{\lambda_{k}}(x, 0) y_{k}(x), x \in \mathbb{T} .
$$

Proof. Similar to [22, Theorem 4].

Lemma 2.3. 10] Let $\left(A_{1}\right)$ holds, then for each $(\tau, \eta) \in \mathbb{T}_{+} \times \mathbb{R}^{n}$ the IVP (2.3) has a unique solution:

$$
a(x)=G_{A}(x, \tau) \eta, x \geq \tau .
$$

Corollary 2.4. 10] Let $\left(A_{1}\right)$ holds, then

$$
\left\|G_{A}(x, \tau)\right\| \leqslant \prod_{\tau<x_{k} \leq x}\left(1+\left\|B_{k}\right\|\right) e^{\|A\|(x-s)}
$$

for $\tau, x \in \mathbb{T}_{+}$with $x \geq \tau$. Moreover, for any $\tau, x \in \mathbb{T}_{+}$, there exists $M>0$ such that we have $\left\|G_{A}(r, s)\right\| \leq M$ on the set

$$
\left\{(r, s) \in \mathbb{T}_{+} \times \mathbb{T}_{+} ; \tau \leq s \leq r \leq x\right\} .
$$

Lemma 2.5. 10 Let $\left(A_{1}\right)$ holds, then

(i) $G_{A}(x, x)=I, x \geq 0$

(ii) $G_{A}(x, s)=G_{A}^{-1}(s, x), 0 \leq s \leq x$;

(iii) $G_{A}(\sigma(x), s)=[I+\mu(x) A] G_{A}(x, s), 0 \leq s \leq x$;

(iv) $G_{A}(x, s) G_{A}(s, r)=G_{A}(x, r), 0 \leq r \leq s \leq x$;

(v) $\frac{\partial}{\Delta s} G_{A}(x, s)=-G_{A}(x, \sigma(s)) A, s \neq x_{k}$

(vi) $G_{A}^{-1}(x, s)=G_{\ominus A^{T}}^{T}(x, s), 0 \leq s \leq x$.

Consider the following nonhomogenous IVP

$$
\left\{\begin{array}{l}
a^{\Delta}=A a+h(x), x \in \mathbb{T}_{+}, x \neq x_{k}, \\
a\left(x_{k}^{+}\right)=\left(I+B_{k}\right) a\left(x_{k}\right), k=1,2, . . \\
a\left(\tau^{+}\right)=\eta, \tau \geqslant 0,
\end{array}\right.
$$

where $h: \mathbb{T}_{+} \rightarrow \mathbb{R}^{n}$ is a given function.

Lemma 2.6. 10 Let $\left(A_{1}\right)$ holds with $h \in \mathcal{R}\left(\mathbb{T}_{+}, \mathbb{R}^{n}\right)$, then for each $(\tau, \eta) \in \mathbb{T}_{+} \times$ $\mathbb{R}^{n}$, the IVP 2.5) has a unique solution:

$$
a(x)=G_{A}(x, \tau) \eta+\int_{\tau}^{x} G_{A}(x, \sigma(s) h(s) \Delta s, x \geq \tau .
$$

Moreover for each $(\tau, \eta) \in \mathbb{T}_{+} \times \mathbb{R}^{n}$, the IDS

$$
\left\{\begin{array}{l}
a^{\Delta}=-A^{T} a^{\sigma}+h(x), x \geq \tau, x \neq x_{k} \\
a\left(x_{k}^{+}\right)=\left(I+C_{k}\right) a\left(x_{k}\right), k=1,2, \cdots \\
a\left(\tau^{+}\right)=\eta, \tau \geqslant 0
\end{array}\right.
$$

has at most one solution, and

$$
a(x)=G_{\ominus A^{T}}(x, \tau) \eta+\int_{\tau}^{x} G_{\ominus A^{T}}(x, s) h(s) \Delta s \quad x \geq \tau
$$

where $C_{k}:=-B^{T}\left(I+B_{k}^{T}\right)^{-1}, k=1,2, \cdots$. 


\section{Diснотому}

The goal of the section is to study the dichotomy of the solution of linear impulsive dynamic systems.

Definition 3.1. [10, Definition 5.1] The IDS 2.3) is said to be exponentially stable if $(\exists) \lambda>0$ with $-\lambda \in \mathcal{R}\left(\mathbb{T}_{+}, \mathbb{R}\right)$ : for every $\tau \in \mathbb{T}_{+},(\exists) N=N(\tau) \geq 1$ : the solution of (2.3) through $(\tau, a(\tau))$ satisfies

$$
\|a(x)\| \leq N\|a(\tau)\| e_{-\lambda}(x, \tau) \text { for all } x \in \mathbb{T}_{+} .
$$

Using the idea of [14, here first we decompose $\mathbb{C}$ into three parts and then with the help of this decomposition we decompose $\mathbb{C}^{m}$ into three spectral subspaces which will help us to use the idea of dichotomy.

For further discussion, we have to divide $\mathbb{C}$ into three sets as follows.

$$
\begin{aligned}
& E_{\mathbb{C}}(\mathbb{T})=\left\{\lambda \in \mathbb{C}: \lim \sup _{S \rightarrow \infty} \frac{1}{S-s_{0}} \int_{s_{0}}^{S} \lim _{u \rightarrow \mu(s)} \frac{\log |1+u \lambda|}{s} \Delta s<0\right\}, \\
& E_{\mathbb{C}}^{+}(\mathbb{T})=\left\{\lambda \in \mathbb{C}: \lim \sup _{S \rightarrow \infty} \frac{1}{S-s_{0}} \int_{s_{0}}^{S} \lim _{u \rightarrow \mu(s)} \frac{\log |1+u \lambda|}{s} \Delta s>0\right\}, \\
& E_{\mathbb{C}}^{0}(\mathbb{T})=\left\{\lambda \in \mathbb{C}: \lim \sup _{S \rightarrow \infty} \frac{1}{S-s_{0}} \int_{s_{0}}^{S} \lim _{u \rightarrow \mu(s)} \frac{\log |1+u \lambda|}{s} \Delta s=0\right\} .
\end{aligned}
$$

Clearly, $\mathbb{C}=E_{\mathbb{C}}(\mathbb{T}) \cup E_{\mathbb{C}}^{+}(\mathbb{T}) \cup E_{\mathbb{C}}^{0}(\mathbb{T})$. By using this decomposition of $\mathbb{C}$, we can state the following definition.

Definition 3.2. Let $\sigma(A)$ denote the spectrum of the regressive matrix $A$. The system $z^{\Delta}(s)=A z(s) ; z\left(s_{0}\right)=z_{0}$ is said to be exponentially stable if all eigenvalues of matrix $A$ are uniformly regressive and $\sigma(A) \subset E_{\mathbb{C}}(\mathbb{T})$ and it is said to be expansive if $\sigma(A) \subset E_{\mathbb{C}}^{+}(\mathbb{T})$. If $\sigma(A) \cap E_{\mathbb{C}}^{0}(\mathbb{T})=\phi$, then the system is said to be dichotomic.

More explicitly the decomposition of $\mathbb{C}^{m}=\bigoplus_{j=1}^{k} \mathcal{L}_{j}$ can be written as

$$
\mathbb{C}^{m}=W_{s}(A) \oplus W_{0}(A) \oplus W_{u}(A),
$$

where

$$
\begin{aligned}
& W_{s}(A)=\bigoplus_{j=1, \lambda_{j} \in E_{\mathbb{C}}(\mathbb{T})}^{k} \operatorname{ker}\left(A-\lambda_{j} I\right)^{n_{j}} \\
& W_{0}(A)=\bigoplus_{j=1, \lambda_{j} \in E_{\mathbb{C}}^{0}(\mathbb{T})}^{k} \operatorname{ker}\left(A-\lambda_{j} I\right)^{n_{j}}
\end{aligned}
$$

and

$$
W_{u}(A)=\bigoplus_{j=1, \lambda_{j} \in E_{\mathbb{C}}^{+}(\mathbb{T})}^{k} \operatorname{ker}\left(A-\lambda_{j} I\right)^{n_{j}}
$$

Now if $A$ is a dichotomic matrix, then $W_{0}(A)=\{0\}$ and so $\mathbb{C}^{m}=W_{s}(A) \oplus W_{u}(A)$. 
Lemma 3.3. 10] Let $\left(A_{2}\right)$ holds. If the solution of IVP

$$
\left\{\begin{array}{l}
a^{\Delta}=A a, x \in \mathbb{T}_{\tau}, x \neq x_{k} \\
a\left(x_{k}^{+}\right)=\left(I+B_{k}\right) a\left(x_{k}\right), k=1,2, \cdots \\
a\left(\tau^{+}\right)=\eta, \tau \geqslant 0
\end{array}\right.
$$

is bounded, then $(\exists) N=N(\tau) \geq 1, \lambda$ with $-\lambda \in \mathcal{R}^{+}$:

$$
\left\|G_{A}(x, \tau)\right\| \leq N e_{-\lambda}(x, \tau)
$$

for all $x \in \mathbb{T}_{(\tau)}$.

Corollary 3.4. [10] Let $\left(A_{2}\right)$ hold, then bounded solution of (3.1) implies that the solution of (2.3) is exponential stable.

Corollary 3.5. 10] Let $\left(A_{2}\right)$ holds, and there exists b, such that

$$
\sup _{k \geq 1}\left\|B_{k}\right\| \leq b, k=1,2, \cdots .
$$

If the solution of IVP (3.1) is bounded, then $\exists$ constants $N, \lambda$ with $-\lambda \in \mathcal{R}^{+}$:

$$
\left\|G_{A}(x, \tau)\right\| \leq N e_{-\lambda}(x, \tau) \text { for all } x \in \mathbb{T}_{(\tau)} .
$$

Lemma 3.6. 3 For any constant $\lambda$ with $-\lambda \in \mathcal{R}^{+}$, we have that

$$
e_{-\lambda}(x, \tau) \leq e^{-\lambda(x-\tau)} \text {, for all } \tau, x \in \mathbb{T} \text { with } x \in \mathbb{T}_{(\tau)} \text {. }
$$

Theorem 3.7. Let $\left(A_{2}\right)$ holds with $A B_{k}=B_{k} A$ and $\mathcal{L}_{j}$ is $\left(I+B_{k}\right)$-invariant subspace of $\mathbb{C}^{m}$ for each $k=1,2,3, \cdots$. Then the system

$$
\left\{\begin{array}{l}
a^{\Delta}=A a, \\
a\left(x_{k}^{+}\right)=\left(I+B_{k}\right) a\left(x_{k}\right), \\
a(0)=0
\end{array}\right.
$$

is exponentially stable if and only if, $b \in \mathbb{C}^{m}$ and each bounded function $f$ the unique solution of the Cauchy problem

$$
\left\{\begin{array}{l}
a^{\Delta}=A a+f(s) b, \\
a\left(x_{k}^{+}\right)=\left(I+B_{k}\right) a\left(x_{k}\right), \\
a(0)=0
\end{array}\right.
$$

is bounded.

Proof. Let $A \in \mathcal{R}\left(\mathbb{T}_{+}, M_{n}(\mathbb{R})\right)$ and $B_{k} \in M_{n}(\mathbb{R})$. Consider the system

$$
\left\{\begin{array}{l}
a^{\Delta}(x)=A a(x) \\
a\left(x_{k}^{+}\right)=\left(I+B_{k}\right) a\left(x_{k}\right), a(0)=0
\end{array}\right.
$$

is exponentially stable. Then by Definition 3.1, we have

$$
\|a(x)\| \leq \alpha\left\|a_{0}\right\| e_{-\gamma}\left(x, x_{0}\right),
$$

for all $x, x_{0} \in \mathbb{T}$. We need to prove that for each $b \in \mathbb{C}^{m}, 3.5$ is bounded. Then from Theorem 2.6, the system 3.5 has atmost one solution with

$$
a(x)=\int_{0}^{x} G_{A}(x, \sigma(s)) f(s) b \Delta s .
$$


Applying norm on equation (3.6), we have

$$
\begin{aligned}
& \|a(x)\|=\sup _{x \in \mathbb{T}} \mid \int_{0}^{x} G_{A}\left(x, \sigma(s) f(s) b \Delta s\left|\leq \sup _{x \in \mathbb{T}}\right| \int_{0}^{x} G_{A}(x, \sigma(s) \Delta s \mid\|f(s) b\|\right. \\
& =\sup _{x \in \mathbb{T}} \mid A^{-1} \int_{0}^{x} A G_{A}\left(x, \sigma(s) \Delta s\left|C=C A^{-1} \sup _{x \in \mathbb{T}}\right| G_{A}(x, 0)-I \mid\right. \\
& \leq C A^{-1}\left\|G_{A}(x, 0)-I\right\| \leq C A^{-1}\left\|G_{A}(x, 0)\right\|+C A^{-1} \\
& \leq C A^{-1} \alpha\left\|a_{0}\right\| e_{-\gamma}(x, 0)+C A^{-1} \\
& \leq C A^{-1} \alpha\left\|a_{0}\right\| e^{-\gamma x}+C A^{-1} .
\end{aligned}
$$

It follows $a(\cdot)$ is bounded.

Sufficiency: Suppose to the contrary that system (3.4) is not exponentially stable. Then for $\nu \in\{1,2, \cdots, k\}$ there exist an eigenvalue $\lambda_{\nu}$ of $A$ such that $\lambda_{\nu} \notin \mathbb{E}_{\mathbb{C}}(\mathbb{T})$. From [22, Remark 1], Thus $\operatorname{Re}\left(\lambda_{\nu}\right) \geq 0$ which implies that $\left|1+u \lambda_{\nu}\right| \geq 1$ for all non-negative $u \in \mathbb{R}$. Letting $f(x)=C$ and $b=0+0+\cdots+0+b_{v}+0+\cdots+0$, then by spectral decomposition theorem implies that

$$
G_{A}(s, \sigma(x)) b=e_{\lambda_{\nu}}(x, \sigma(s)) p_{\nu}(s)
$$

where $p_{\nu}(s)$ is a polynomial of degree less than or equal to $m_{\nu}-1$. In this solution of (3.5) can be written as

$$
\begin{aligned}
& a(x)=\int_{0}^{x} G_{A}(x, \sigma(s) f(s) b \Delta s \\
& =\int_{0}^{x} e_{\lambda_{\nu}}\left(x, \sigma(s) p_{\nu}(s) C \Delta s\right. \\
& =C \int_{0}^{x} \frac{e_{\lambda \nu}(x, s)}{1+\mu \lambda_{\nu}} p_{\nu}(s) \Delta s
\end{aligned}
$$

Since $\operatorname{Re}\left(\lambda_{\nu}\right) \geq 0$, so $a(x) \rightarrow \infty$ as $x \rightarrow \infty$ that is unique solution of (3.5) is unbounded, which is a contradiction. Hence the system (3.4) is exponentially stable.

Example 3.8. Let us consider the IVP given in Example 2.1 with $A=\left(\begin{array}{cc}-\lambda_{1} & 0 \\ 0 & -\lambda_{2}\end{array}\right)$, $\left(I+B_{k}\right)=\left(\begin{array}{cc}a_{2 k} & 0 \\ 0 & b_{2 k}\end{array}\right), \lambda_{2}>\lambda_{1}>0$ and $x_{k}:=k$. Moreover, we choose $\left(a_{2 k}\right)_{k \geq 1}$ and $\left(b_{2 k}\right)_{k \geq 1}$ such that $a_{i j}:=a_{2(i+1)} a_{2(i+2)} \cdots a_{2(i+j)} \leq a$ and $b_{i j}:=$ $b_{2(i+1)} b_{2(i+2)} \cdots b_{2(i+j)} \leq b$ for each fixed $i \geq 0$ and for $j=1,2, \cdots$.

It is easy to see that $A B_{k}=B_{k} A$ and $\mathcal{L}_{j}$ is $\left(I+B_{k}\right)$-invariant subspace of $\mathbb{C}^{m}$ for each $k=1,2,3, \cdots$, and for $0<\theta<1, x_{k+1}-x_{k}<\theta, k=1,2, \cdots$. It implies that for $b \in \mathbb{C}^{m}$ and each bounded function $f$ the unique solution of the Cauchy problem

$$
\left\{\begin{array}{l}
a^{\Delta}=\left(\begin{array}{cc}
-\lambda_{1} & 0 \\
0 & -\lambda_{2}
\end{array}\right) a+f(s) b, x \in \mathbb{T}_{+}, x \neq x_{2 k} \\
a\left(x_{2 k}^{+}\right)=\left(\begin{array}{cc}
a_{2 k} & 0 \\
0 & b_{2 k}
\end{array}\right) a\left(x_{2 k}\right), k=1,2, \cdots \\
a(0)=0, \tau \geqslant 0 .
\end{array}\right.
$$




$$
\begin{aligned}
& a(x)=\int_{0}^{x} G_{A}(x, \sigma(s)) f(s) b \Delta s \text { is bounded, where }
\end{aligned}
$$

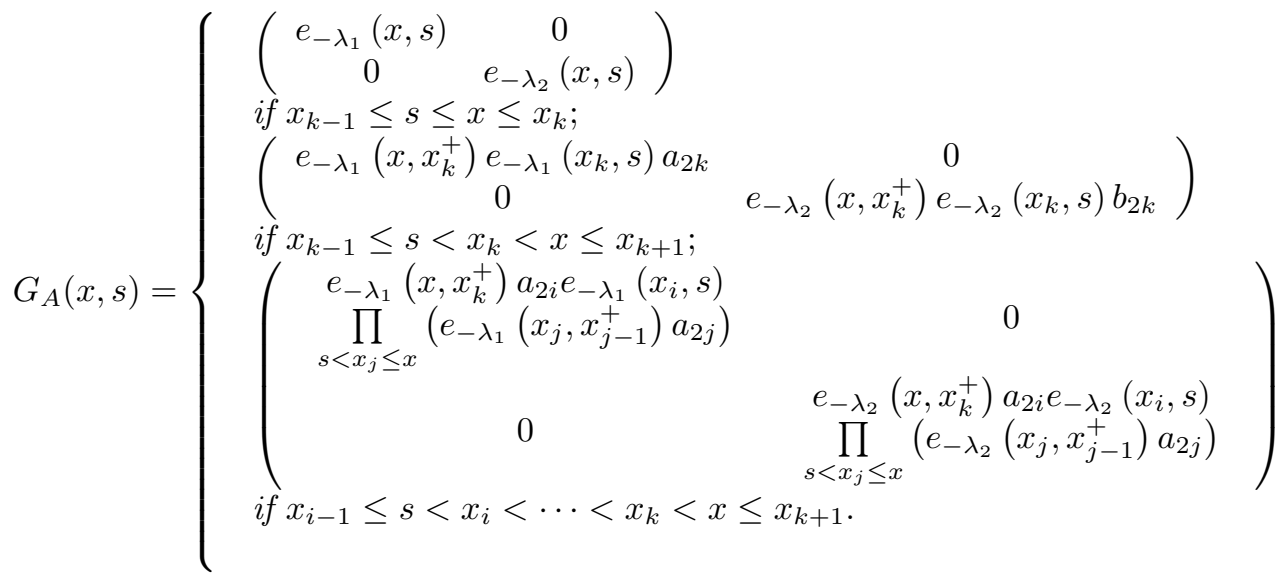

From Theorem 3.7, it follows that the system

$$
\left\{\begin{array}{l}
a^{\Delta}=\left(\begin{array}{cc}
-\lambda_{1} & 0 \\
0 & -\lambda_{2}
\end{array}\right) a, x \neq x_{2 k} \\
a\left(x_{2 k}^{+}\right)=\left(\begin{array}{cc}
a_{2 k} & 0 \\
0 & b_{2 k}
\end{array}\right) a\left(x_{2 k}\right), k=1,2, \cdots \\
a(0)=0
\end{array}\right.
$$

is exponentially stable

Theorem 3.9. Suppose that $B_{k} \in M_{n}(\mathbb{R}), k=1,2, \cdots, A \in \mathcal{R}\left(\mathbb{T}_{+}, M_{n}(\mathbb{R})\right)$ $A B_{k}=B_{k} A$ and $\mathcal{L}_{j}$ is $\left(I+B_{k}\right)$-invariant subspace of $\mathbb{C}^{m}$ for each $k=1,2,3, \cdots$, and there exist a positive constant $\theta$ such that $x_{k+1}-x_{k}<\theta, k=1,2, \cdots$. Then the system (3.4) is dichotomic if and only if there exists a projection $Q$ having the property

$$
G_{A}(x, 0) Q=Q G_{A}(x, 0),
$$

for all $x \geq 0$ such that for each non-zero vector $b \in \mathbb{C}^{m}$ and each bounded function $f$ such that $G_{A}(s, 0) f(s)=f(s) G_{A}(s, 0)$, the solutions of the following Cauchy problems

$$
\left\{\begin{array}{l}
a^{\Delta}=A a+f(s) Q b \\
a\left(x_{k}^{+}\right)=a\left(x_{k}^{-}\right)+B_{k} a\left(x_{k}\right) \\
a(0)=0
\end{array}\right.
$$

and

$$
\left\{\begin{array}{l}
a^{\Delta}=-A a^{\sigma}+f(s)(I-Q) b \\
a\left(x_{k}^{+}\right)=a\left(x_{k}^{-}\right)+B_{k} a\left(x_{k}\right) \\
a(0)=0
\end{array}\right.
$$

are bounded.

Proof. Necessity: Suppose that $A$ is dichotomic. By [22, Remark 2] $\mathbb{C}^{m}=W_{s}(A) \oplus$ $W_{\mu}(A)$. Let us define $Q: \mathbb{C}^{m} \rightarrow \mathbb{C}^{m}$ by $Q(w):=w_{s}$, where $w=w_{s}+w_{\mu}, w_{s} \in W_{s}$ and $w_{\mu} \in W_{\mu}$. Clearly $Q$ is a projection. Moreover for all $w \in \mathbb{C}^{m}$ and all $s \geq 0$, 
we have

$$
\begin{aligned}
& Q G_{A}(s, 0) w=Q G_{A}(s, 0)\left(w_{s}+w_{\mu}\right) \\
& =Q\left(G_{A}(s, 0) w_{s}+G_{A}(s, 0) w_{\mu}\right) \\
& =G_{A}(s, 0) w_{s} \\
& =G_{A}(s, 0) Q_{w},
\end{aligned}
$$

where the fact that $W_{s}(A)$ is an $G_{A}(s, 0)$-invariant subspace, thus

$$
Q G_{A}(s, 0) w=G_{A}(s, 0) Q w .
$$

Now the solution of 3.8 is given by

$$
a(x)=\int_{0}^{x} G_{A}(x, \sigma(s)) f(s) Q b \Delta s .
$$

From equation 3.10 , we consider $G_{A}(x, \sigma(s)) f(s) Q b$, we can write

$$
\begin{aligned}
& G_{A}(x, \sigma(s)) f(s) Q b=f(s) G_{A}(x, \sigma(s)) Q b \\
& =f(s) Q\left(e_{\lambda_{1}}(x, \sigma(s)) p_{1}(s)+e_{\lambda_{2}}(x, \sigma(s)) p_{2}(s)\right. \\
& +\cdots+e_{\lambda_{\nu}}(x, \sigma(s)) p_{\nu}(s)+\cdots+e_{\lambda_{k}}(x, \sigma(s)) p_{k}(s) \\
& =f(s) Q\left(e_{\lambda_{1}}(x, \sigma(s)) p_{1}(s)+e_{\lambda_{2}}(x, \sigma(s)) p_{2}(s)\right. \\
& +\cdots+e_{\lambda_{\nu}}(x, \sigma(s)) p_{\nu}(s),
\end{aligned}
$$

where $\lambda_{i} \in W_{s}(A)$ for $i \in\{1,2, \cdots, \nu\}$ and $\lambda_{j} \in W_{\mu}(A)$ for $j \in\{1,2, \cdots, k\}$. It is clear that the solution of $(3.8)$ is bounded.

Sufficiency: Suppose to the contrary that $A$ is not dichotomic. Then $W_{0}(A) \neq 0$ and so there exists an eigenvalue $\lambda_{l}$ of $A$ such that $\lambda_{l} \in \mathbb{E}_{\mathbb{C}}^{0}(\mathbb{T})$. Since $b \in \mathbb{C}^{m}$, so letting $b=0+0+0+\cdots b_{l}+0+0$, then either $b_{l} \in W_{s}(A)$ or $b_{l} \in W_{\mu}(A)$. If $l \leq v$, then $b_{l} \in W_{s}(A)$ and if $l>v$, then $b_{l} \in W_{\mu}(A)$.

Case 1. Assume $b_{l} \in W_{s}(A)$. Then by Lemma 2.2, we have

$$
\begin{aligned}
& G_{A}(x, \sigma(s)) f(s) Q b \\
& =G_{A}(x, \sigma(s)) f(s) Q b_{l} \\
& =f(s) e_{\lambda_{l}}(x, \sigma(s)) p_{l}(s),
\end{aligned}
$$

where $p_{l}(\cdot)$ is a polynomial of degree less than or equal to $m_{l}-1$. So, the solution of 3.8 becomes

$$
a(x)=\int_{0}^{x} G_{A}(x, \sigma(s) f(s) Q b \Delta s,
$$

where $p_{l}(\cdot)$ is at most $m_{l}-1$ degree polynomial. Let $f(s)=C$, then equation (3.11 becomes

$$
a(x)=\int_{0}^{x} C e_{\lambda_{l}}(x, \sigma(s)) p_{l}(s) \Delta s
$$

or

$$
a(x)=C \int_{0}^{x} e_{\lambda_{l}}(x, \sigma(s)) p_{l}(s) \Delta s .
$$

Since $\operatorname{Re}\left(\lambda_{\nu}\right) \geq 0$, so $a(x) \rightarrow \infty$ as $x \rightarrow \infty$ that is unique solution of (3.8) is unbounded, which is contradiction. Hence the system $A$ is dichotomic.

Case2: Suppose $b_{l} \in W_{\mu}(A)$ So the solution of $(3.9)$ is given by

$$
w(x)=\int_{0}^{x} G_{\ominus A}(x, s) f(s)(I-Q) b \Delta s .
$$


By Lemma 2.2, we have

$$
\begin{aligned}
& G_{\ominus A}(x, \sigma(s)) f(s)(I-Q) b \\
& =G_{\ominus A}(x, \sigma(s)) f(s)(I-Q) b_{l} \\
& =f(s) e_{\ominus A}(x, \sigma(s))(I-Q) b_{l} \\
& =f(s) e_{\ominus \lambda_{l}}(x, \sigma(s)) p_{l}(s),
\end{aligned}
$$

where $p_{l}(s)$ is a polynomial of degree less than or equal to $m_{l}-1$. So, the solution of $(3.9)$ becomes

$$
w(x)=\int_{0}^{x} f(s) e_{\ominus \lambda_{l}}(x, \sigma(s)) p_{l}(s) \Delta s,
$$

Let $f(s)=C$, then equation 3.12 becomes

$$
w(x)=\int_{0}^{x} C e_{\ominus \lambda_{l}}(x, \sigma(s)) p_{l}(s) \Delta s
$$

or

$$
w(x)=C \int_{0}^{x} e_{\ominus \lambda_{l}}(x, \sigma(s)) p_{l}(s) \Delta s .
$$

Clearly $e_{\ominus \lambda_{l}}(x, \sigma(s)) \rightarrow 1$ as $x \rightarrow \infty$ so, $w(x) \rightarrow \infty$ as $x \rightarrow \infty$ that is unique solution of $(3.9)$ is unbounded, which is a contradiction. Hence in this case we also arrived at a contradiction the system (3.4) is dichotomic. The proof is complete.

\section{Conclusion}

We have presented some new criteria for admitting the exponential dichotomy of impulsive dynamic systems. If the assumption of $\left(I+B_{k}\right)$-invariant subspace of $\mathbb{C}^{m}$ is dropped this can be an open problem to formulate Spectral Decomposition Theorem for impulsive transition matrix $G_{A}(x, s), 0 \leq s \leq x$, associated with $\left\{B_{k}, x_{k}\right\}_{k=1}^{\infty}$.

This topic warrants further investigation. In particular, it should be established connections between the Hyers-Ulam stability of the linear impulsive dynamic systems and its dichotomy. Also, it would be interesting to know the conditions for Hyers-Ulam stability.

\section{Declarations}

Funding. Not applicable.

Availability of data and materials. Not applicable.

Competing interests. The authors declare that they have no competing interests. Authors' contributions. The authors have contributed equally to this manuscript. They read and approved the final manuscript.

Acknowledgments. The authors would like to thank the anonymous referee for his/her comments that helped us improve this article.

\section{REFERENCES}

[1] A. Alonso, J. L. Hong, R. Obaya, Exponential dichotomy and trichotomy for difference equations, Computers 8 Mathematics with Applications, (38) (1999) 41-49.

[2] M. Bohner, A. Peterson, Advancs in Dynamics Equations on Time scales, Birkhauser, Boston, 2003.

[3] M. Bohner, A. Peterson, Dynamic Equations on Time Scales: An Introduction with Applications, Birkhauser, Boston, Mass, USA, 2001. 
[4] S. N. Chow, H. Leiva, Existence and roughness of the exponential dichotomy for skew-product semiflows in Banach spaces, Journal of Differential Equations, (120) (1995) 429-477.

[5] W. A. Coppel, Dichotomies in Stability Theory, Lecture Notes in Mathematics, vol. 629. Springer-Verlag: Berlin, New York, 1978.

[6] N. Ju, S. Wiggins, On roughness of exponential dichotomy, Journal of Mathematical Analysis and Applications, 2001; 262:39-49.

[7] V. Lakshmikantham, D. D. Bainov, P. S. Simeonov, Theory of Impulsive Differential Equations, World Scientific, Singapore, 1989.

[8] V. Lupulescu, A. Younus, Controllability and observability for a class of time-varying impulsive systems on time scales, Electron. J. Qual. Theory Differ. Equ., 2011, No. 95, 30 pp.

[9] V. Lupulescu, A. Younus, On controllability and observability for a class of linear impulsive dynamic systems on time scales, Math. Comput. Modelling 54 (2011), no. 5-6, 1300-1310.

[10] V. Lupulescu, A. Zada, Linear impulsive dynamic systems on time scales, Electron. J. Qual. Theory Differ. Equ. 2010, No. 11, 30 pp.

[11] V. Milman, A. Myshkis, On the stability of motion in the presence of impulses, Sib. Math. J., 1 (2) $1960233-7$.

[12] O. Perron, Die stabilitätsfrage bei differentialgleichungen. Mathematische Zeitschrift (32) (1930) 703-728.

[13] C. Pötzsche, Exponential dichotomies for dynamic equations on measure chains. Nonlinear Analysis TMA 2001; 479 873-884.

[14] C. Pötzsche, S. Siegmund, and F. Wirth, A spectral characterization of exponential stability for liner time-invariant systems on time scales, Discrete Contin. Dyn. Syst., 9 (2003), 12231241.

[15] A. R. Seadawy, M. Iqbal, D. Lu., Nonlinear wave solutions of the Kudryashov-Sinelshchikov dynamical equation in mixtures liquid-gas bubbles under the consideration of heat transfer and viscosity. J. Taibah Univ. Sci. 2019;13(1):1060-1072.

[16] A. R. Seadawy, D. Lu, M. Iqbal. Application of mathematical methods on the system of dynamical equations for the ion sound and Langmuir waves. Pramana J Phys. 93 (2019), $1-10$.

[17] V. I. Slyn'ko, C. Tunc. Stability of abstract linear switched impulsive differential equations. Automatica 107 (2019): 433-441.

[18] V. I. Slyn'ko, C. Tunc. Global asymptotic stability of nonlinear periodic impulsive equations. Miskolc Mathematical Notes. 19(1) (2018), 595-610.

[19] V. I. Slyn'ko, C. Tunc. Instability of set differential equations. Journal of Mathematical Analysis and Applications 467.2 (2018), 935-947.

[20] Y. Wang, J. Lu, Some recent results of analysis and control for impulsive systems, Commun Nonlinear Sci Numer Simulat, 80 (2019) 104862.

[21] A. Younus, M. Asif, Input distinguishability of linear dynamic control systems. J. Taibah Univ. Sci. 2019;13(1),1100-1107.

[22] A. Zada, T. Li, S. Ismail, O. Shah, Exponential dichotomy of linear autonomous systems over time scales. Differ. Equ. Appl. 8 (2016), no. 2, 123-134.

Gulnaz Atta

Department of Mathematics, University of Education, D. G. Khan, PakistanDepartment of Mathematics, University of Education, D. G. Khan, Pakistan

E-mail address: uegulnaz@gmail.com

Awais Younus

CASPAM, Bahauddin Zakaryia University, Multan, Pakistan

E-mail address: awaissms@yahoo.com 